Original Research Paper

\title{
Solutions of the Duffing and Painlevé-Gambier Equations by Generalized Sundman Transformation
}

\author{
${ }^{1}$ Damien Kolawolé Kêgnidé Adjaï, ${ }^{1}$ Lucas Hervé Koudahoun, ${ }^{1}$ Jean Akande, \\ ${ }^{2}$ Yélomè Judicaël Fernando Kpomahou and ${ }^{1}$ Marc Delphin Monsia \\ ${ }^{I}$ Department of Physics, University of Abomey-Calavi, Abomey-Calavi, 01. BP.526, Cotonou, Benin \\ ${ }^{2}$ Department of Industrial and Technical Sciences, ENSET-Lokossa, University of Abomey, Abomey, Benin
}

Article history

Received: 20-03-2018

Revised: $16-06-2018$

Accepted: 03-11-2018

Corresponding Author:

Damien K.K. Adjaï

Department of Physics,

University of Abomey-Calavi,

Abomey-Calavi, 01. BP.526,

Cotonou, Benin

Email: adjkolakegni@gmail.com

\begin{abstract}
A new approach using the generalized Sundman transformation to solve explicitly and exactly in a straightforward manner the cubic elliptic Duffing equation is proposed in this study. The method has the advantage to closely relate this equation to the linear harmonic oscillator equation and to be applied to solve other nonlinear differential equations. As a result, explicit and exact general periodic solutions to some PainlevéGambier type equations have been established and in particular, it is shown that a reduced Painlevé-Gambier XII equation can exhibit trigonometric solutions, but with a shift factor.
\end{abstract}

Keywords: Cubic Duffing Equation, Painlevé-Gambier Equations, Jacobian Elliptic Functions, Exact Periodic Solution, Generalized Sundman Transformation

\section{Introduction}

A large part of physics, engineering and applied science problems is solved by using linear models where the principle of superposition may be applied. However, this consideration is only valid as a first approximation, since a more realistic approach in the mathematical modeling of many processes and phenomena in physics and applied sciences leads to consider nonlinear problems often expressed in terms of nonlinear differential equations. The phenomena of oscillation, dielectric polarization, creep deformation and stress relaxation under loading in dynamic structures are examples of problems where the nonlinearity properties occur in different aspects (Kpomahou and Monsia, 2015). In structural dynamics, for example, geometric nonlinearities are often due to large displacements or large deformations inducing not only stiffening but also nonlinear optical properties of the structure. The material nonlinearity is relative to the stress-strain constitutive law which implies the phenomena of dissipation of energy in heat and the inertial nonlinearity results often from the quadratic terms in the first order derivative in the equations of motion or deformation (Kpomahou and Monsia, 2015; Kerschen et al., 2006). Thus, the failure to take into account these nonlinearities can permanently affect the performance of these material structures during their service, which can become a danger for users and the environment. In this sense, good design of these structures or repair after damage requires to calculate the explicit and exact general solutions of the nonlinear differential equations describing their dynamics. In this perspective the explicit and exact general solutions of these nonlinear differential equations are more appropriate from the physical point of view as well as engineering application viewpoint than approximate and numerical solutions. In so doing, the periodic solutions constitute an important class of solutions since many phenomena in the real world, such as mechanical oscillations of conservative systems, are of a periodic nature. Thus a special interest must be accorded to the explicit and exact general periodic solutions. However, it should be emphasized that there is no master mathematical method that works in all cases of calculation of explicit and exact general periodic solutions. It suffices to note the large and rich variety of mathematical methods existing in the literature for this purpose. Therefore the fundamental problem of finding explicit and exact general periodic solutions to nonlinear differential equations constitutes yet an active research field of mathematics. It is also known that such solutions are only possible for a few number of nonlinear differential equations. There appears then logic to look for mathematical theories which may provide in a straightforward manner explicit and exact general periodic solutions to nonlinear differential equations. In 
the process of solving nonlinear second order differential equations, it is convenient to consider linearization methods which transform the initial equation into a linear differential equation whose properties are well known. As such, one can note methods like point transformation, contact transformation and generalized Sundman transformation. The latter, according to Euler and Euler (2004), is a more general transformation than the first two transformations of linearization. The generalized Sundman transformation is a nonlocal transformation that has been frequently used to determine the first integrals and solutions of a variety of Liénard-type differential equations (Euler and Euler, 2004; Partha et al., 2010; Nakpim and Meleshko, 2010). Recently, Akande et al. (2017a) have formulated a generalized Sundman transformation applied to the harmonic oscillator equation to demonstrate for the first time, the existence of a class of quadratic Liénard type equations whose solutions are trigonometric functions, but with amplitude dependent frequency. Thus, it has been shown that equation XVIII of the Painlevé-Gambier classification (Ince, 1956) and its inverted version and other quadratic Liénard type equations admit trigonometric solutions by applying the generalized Sundman transformation developed. It is also well known that the study of oscillation in many mechanical systems leads to consider the cubic elliptic Duffing equation consisting to introduce the cubic term in the displacement into the harmonic oscillator equation of the linear structural model in order to take into account the geometric nonlinearity properties. Recently it is found that the theoretical investigation of viscoelastic deformation in material structures taking into consideration simultaneously the geometric, material and inertial nonlinearities, leads to nonlinear problems described in terms of mixed or quadratic Liénard type equations (Monsia, 2012). One may note in this context that the quadratic Liénard type differential equations and the cubic Duffing equation have important applications in physics. The cubic Duffing equation plays an interesting role in many areas of physics like classical and quantum mechanics (Reinhardt and Walker, 2010). In this regard the forced cubic Duffing equation is often, for example, used to illustrate a number of nonlinear system characteristic such as jumps, subharmonic and superharmonic resonances in addition to the amplitude dependent frequency exhibited already by the free cubic Duffing oscillator. Let us now consider the cubic Duffing equation and equations XIX and XII of the PainlevéGambier classification. The general solutions of the unforced cubic Duffing equation are well known in the literature (Lakshmanan and Rajaseekar, 2003). It is also well known that the Jacobian elliptic functions are solutions of this equation. On the other hand, Painlevé- Gambier equations XIX and XII have recently been studied by the application of generalized Sundman transformation
(Partha et al., 2010). The authors (Partha et al., 2010) were able to calculate the first integrals in addition to those already determined by Ince (1956) and parametric solutions for these equations. However, no explicit and exact general periodic solution has been computed for these equations by the generalized Sundman transformation used by those authors (Partha et al., 2010). The cubic Duffing equation belongs to the usual class of Liénard equations, whereas the Painlevé- Gambier XII and XIX equations are of the class of the quadratic Liénard type equations. According to the classification given by Ince (1956), the equation XII is a canonical equation of type II, whereas the equation XIX is a canonical equation of type III. Although these equations have been already studied in the literature with more or less of success, it is important, to further identify their analytical properties, to investigate them by different types of analytical methods, which may be explicitly and exactly applied in a straightforward fashion. In other words, it is reasonable to ask whether other integration methods could not be applied for such equations, since these new methods may appear easy and more straightforward to be applied for solving differential equation and may lead to new solutions in some cases. The problem now is to show that the explicit and exact general periodic solutions can be computed for these nonlinear differential equations by applying the generalized Sundman transformation proposed by Akande et al. (2017a). Such a solving approach is novel since it has never been used in the past to explicitly and exactly calculate the general solution to the cubic Duffing equation and to some Painlevé-Gambier type equations. The fundamental question to be solved, in the context of the generalized Sundman transformation proposed by Akande et al. (2017a), can then be formulated as follows: Can we calculate the explicit and exact general periodic solutions of the equations considered above? The present work supposes this fulfillment by applying the generalized Sundman transformation proposed by Akande et al. (2017a). This fact will allow testing the accuracy and efficiency of the proposed generalized Sundman transformation to ensure the calculation of explicit and exact general periodic solutions to a wide variety of nonlinear ordinary differential equations that are often encountered in mathematical modeling of physics and applied science problems. The present theory will have the advantage of generalizing the approach developed by Akande et al. (2017a) and other methods that exist in literature in the perspective of detecting large class of nonlinear differential equations of the Liénard type for which explicit and exact general periodic solutions can be computed by a well-documented linearization method, that is the generalized Sundman transformation. This theory will have also the advantage to closely relate for 
the first time the Duffing and some Painlevé-Gambier type equations to the linear harmonic oscillator differential equation with well known properties, that is to show their mathematical equivalence. As practical implications, these results may be useful in numerical experiments involving these equations. To compute the explicit and exact general periodic solutions of these equations, a brief review of the theory introduced by Akande et al. (2017a) is given (section 2) and secondly, the general theory under question is carried out (section 3). Finally the explicit and exact general periodic solutions to unforced cubic Duffing equation and for some Painlevé-Gambier type equations are computed (section 4) and, a discussion (section 5) and a conclusion for the research contribution are carried out.

\section{Review of the Theory by Akande and Coworkers (Akande et al., 2017a)}

Let us consider the general second order linear differential equation as:

$$
y^{\prime \prime}(\tau)+b y^{\prime}(\tau)+a^{2} y(\tau)=0
$$

where, prime denotes differentiation with respect to $\tau, a$ and $b$ are arbitrary parameters. By application of the generalized Sundman transformation:

$$
y(\tau)=F(t, x), d \tau=G(t, x) d t, G(t, x) \frac{\partial F(t, x)}{\partial x} \neq 0
$$

with:

$$
F(t, x)=\int g(x)^{l} d x, G(t, x)=\exp (\gamma \varphi(x))
$$

where, $l$ and $\gamma$ are arbitrary parameters and $g(x) \neq 0$ and $\varphi(x)$ are arbitrary functions of $x$, to (1), one may obtain:

$$
\begin{aligned}
& \ddot{x}+\left(l \frac{g^{\prime}(x)}{g(x)}-\gamma \varphi^{\prime}(x)\right) \dot{x}^{2}+b \dot{x} \exp (\gamma \varphi(x)) \\
& +\frac{a^{2} \exp (2 \gamma \varphi(x)) \int g(x)^{l} d x}{g(x)^{l}}=0
\end{aligned}
$$

as general class of mixed Liénard type differential equations where the dot over a symbol means differentiation with respect to time.

The parametric choice $b=0$, leads to:

$$
\begin{aligned}
& \ddot{x}+\left(l \frac{g^{\prime}(x)}{g(x)}-\gamma \varphi^{\prime}(x)\right) \dot{x}^{2} \\
& +\frac{a^{2} \exp (2 \gamma \varphi(x)) \int g(x)^{l} d x}{g(x)^{l}}=0
\end{aligned}
$$

as general class of quadratic Liénard type nonlinear equations. An interesting case of (4) is obtained by considering $l=0$, viz:

$$
\ddot{x}-\gamma \varphi^{\prime}(x) \dot{x}^{2}+a^{2} x \exp (2 \gamma \varphi(x))=0
$$

The importance of (5) is that it exhibits trigonometric functions as exact periodic solutions but with amplitude-dependent frequency. Akande et al. (2017a) it is shown for the first time that some existing Liénard type equations may exhibit exact trigonometric solutions. One may also for example, find that the quadratic Liénard type equation:

$\ddot{x}+\frac{1}{2} \frac{\dot{x}^{2}}{1+x}+\frac{x}{1+x}=0$

used to model oscillation of a liquid column in a U-tube (Utz, 1970) belongs to the class of equations defined by (5) by putting $\varphi(x)=\frac{1}{2} \ln (1+x)$ and $\gamma=-1$, so that this equation may exhibit trigonometric functions as exact periodic solution (Akande et al., 2017b). That being so the general theory under question may be formulated.

\section{General Theory}

The objective of this section is to extend the preceding theory to look for large classes of linearizable Liénard type equations in order to ensure exact solutions which may be expressed explicitly or by quadratures. In the previous theory the general second order linear equation is considered in the form of homogeneous equation that is with no forcing function. Instead in this section, let us consider the general second order linear Equation 1with a constant forcing term, that is:

$y^{\prime \prime}(\tau)+b y^{\prime}(\tau)+a^{2} y(\tau)=c$

where, $c$ is an arbitrary parameter. The application of the generalized Sundman transformation (2) to (7) yields:

$$
\begin{aligned}
& \ddot{x}+\left(l \frac{g^{\prime}(x)}{g(x)}-\gamma \varphi^{\prime}(x)\right) \dot{x}^{2}+b \dot{x} \exp (\gamma \varphi(x)) \\
& +\frac{a^{2} \exp (2 \gamma \varphi(x)) \int g(x)^{l} d x}{g(x)^{l}}-\frac{c \exp (2 \gamma \varphi(x))}{g(x)^{l}}=0
\end{aligned}
$$

The comparison of (3) with (8) shows that the constant forcing function $c$ contributes to the general class of mixed Liénard type Equation 8 by an additional term $\frac{c \exp (2 \gamma \varphi(x))}{g(x)^{l}}$. This establishes the extension of the 
theory by Akande et al. (2017a) to a wider class of Liénard type nonlinear differential equations. The question now is to know: What happens to Equation 4 and 5 with respect to this additional term? Let us consider then $b=0$ and $l=0$. The parametric choice $b=0$ leads to:

$$
\begin{aligned}
& \ddot{x}+\left(l \frac{g^{\prime}(x)}{g(x)}-\gamma \varphi^{\prime}(x)\right) \dot{x}^{2} \\
& +\frac{a^{2} \exp (2 \gamma \varphi(x)) \int g(x)^{l} d x}{g(x)^{l}}-\frac{c \exp (2 \gamma \varphi(x))}{g(x)^{l}}=0
\end{aligned}
$$

The comparison of (4) with (9) shows also the persistence of the preceding additional term in the generalized class of quadratic Liénard type equations represented by (9). The application of $l=0$ to (9) gives the class of quadratic Liénard type equations:

$$
\ddot{x}-\gamma \varphi^{\prime}(x) \dot{x}^{2}+a^{2} x \exp (2 \gamma \varphi(x))-c \exp (2 \gamma \varphi(x))=0
$$

which differs from the class of quadratic Liénard type Equation 5 by the additional term $-c \exp (2 \gamma \varphi(x))$. By noting:

$$
y(\tau)=A_{0} \sin (a \tau+\alpha)+\frac{c}{a^{2}}
$$

where, $a \neq 0$, the general solution to (7) with $b=0$, the general solution to (10) becomes:

$$
x(t)=A_{0} \sin (a \phi(t)+\alpha)+\frac{c}{a^{2}}
$$

where, $A_{0}$ and $\alpha$ are arbitrary parameters and $\tau=\phi(t)$ satisfies:

$$
d t=\exp (-\gamma \varphi(x)) d \phi(t)
$$

In this way the above results may be formulated in terms of the following theorem.

\section{Theorem}

Consider $x(t)$ given by (12). If $\phi(t)$ satisfies (13), then $x(t)$ satisfies (10).

The solution (12) remains of periodic form. For a convenient choice of $\varphi(x)$ and $\gamma$ the solution (12) may exhibit harmonic periodic behavior but with a shift factor $\frac{c}{a^{2}}$. This extends therefore the theory by Akande et al. (2017a). It is now convenient to show the ability of the current theory to provide explicit and exact general periodic solutions to some existing Liénard equations.

\section{Applications of General Theory}

This section is devoted to show the usefulness of the present theory by considering some well known nonlinear equations.

\section{Cubic Duffing Equation}

The cubic Duffing equation is one of the most investigated equations from mathematical standpoint as well as physical viewpoint. The motivation results from the fact that this equation arises in mathematical modeling of many problems of mechanics and quantum mechanics, for example. The cubic Duffing equation is of the form (Nayfeh and Mook, 1979):

$\ddot{x}+\omega_{0}^{2} x+\beta x^{3}=0$

where, $\omega_{0}$ and $\beta$ are arbitrary parameters. From the mechanical viewpoint, the parameter $\beta>0$ is called hardening parameter while $\beta<0$ is called softening parameter and $\beta=0$, gives the well known linear harmonic oscillator equation. The equation (14) may be recovered from the general class of quadratic Liénard Equation 9. Indeed, the functional choice $\varphi(x)=\ln (f(x))$ leads to:

$$
\begin{aligned}
& \ddot{x}+\left(l \frac{g^{\prime}(x)}{g(x)}-\gamma \frac{f^{\prime}(x)}{f(x)}\right) \dot{x}^{2} \\
& +\frac{a^{2} f(x)^{2 \gamma} \int g(x)^{l} d x}{g(x)^{l}}-\frac{c f(x)^{2 \gamma}}{g(x)^{l}}=0
\end{aligned}
$$

which for $f(x)=x^{2}$ and $g(x)=x$, yields:

$$
\ddot{x}+(l-2 \gamma) \frac{\dot{x}^{2}}{x}+\frac{a^{2}}{l+1} x^{4 \gamma+1}-c x^{4 \gamma-l}=0
$$

The parametric choice $l=2 \gamma=1$, reduces (16) to:

$$
\ddot{x}-c x+\frac{a^{2}}{2} x^{3}=0
$$

which is the Duffing Equation 14 by noting $c=-\omega_{0}^{2}$ and $\frac{a^{2}}{2}=\beta$. So the general solution to the Duffing Equation 14 may be written according to (12):

$$
x(t)=\varepsilon \sqrt{-\frac{\omega_{0}^{2}}{\beta}+2 A_{0} \sin (a \phi(t)+\alpha)}
$$

where:

$$
\varepsilon d(t)=\frac{d \phi(t)}{\sqrt{-\frac{\omega_{0}^{2}}{\beta}+2 A_{0} \sin (a \phi(t)+\alpha)}}
$$


and $\varepsilon= \pm 1$. The evaluation of the integral resulting from (19):

$$
J=\int \frac{d \phi(t)}{\sqrt{-\frac{\omega_{0}^{2}}{\beta}+2 A_{0} \sin (a \phi(t)+\alpha)}}
$$

depends on the value of $\omega_{0}^{2}$ and $\beta$. Therefore three distinct cases may be considered in this study (Gradshteyn and Ryzhik, 2007).

Case 1: $\beta>0$ and $\omega_{0}^{2}<0$

In this case where $\beta>0$ and $\omega_{0}^{2}<0$, it is moreover assumed that $-\frac{\omega_{0}^{2}}{\beta}>2 A_{0}>0$. By noting (Gradshteyn and Ryzhik, 2007):

$$
p=2 \sqrt{\frac{A_{0}}{-\frac{\omega_{0}^{2}}{\beta}+2 A_{0}}}
$$

that is:

$$
p=2 \sqrt{\frac{\beta A_{0}}{2 \beta A_{0}-\omega_{0}^{2}}}
$$

and:

$$
\delta=\arcsin \left(\sqrt{\frac{1-\sin (a \phi+\alpha)}{2}}\right)
$$

the integral $J$ becomes (Gradshteyn and Ryzhik, 2007):

$$
J=-\frac{1}{a} \frac{p}{\sqrt{A_{0}}} F(\delta, p)
$$

where, $F(\delta, k)$ is the elliptic integral of the first kind so that using (19):

$$
\sin \delta=\operatorname{sn}\left(-\frac{a \varepsilon \sqrt{A_{0}}}{p}(t+C), p\right)
$$

where, $\operatorname{sn}(z, k)$ designates the Jacobian elliptic function and $C$ an arbitrary parameter. In this way:

$$
\sqrt{\frac{1-\sin (a \phi+\alpha)}{2}}=\operatorname{sn}\left(-\frac{a \varepsilon \sqrt{A_{0}}}{p}(t+C), p\right)
$$

that is to say:

$$
\sin (a \phi+\alpha)=1-2 s n^{2}\left(-\frac{a \varepsilon \sqrt{A_{0}}}{p}(t+C), p\right)
$$

from which the general solution to the Duffing Equation 14 becomes:

$$
x(t)=\varepsilon\left[\frac{-\omega_{0}^{2}}{\beta}+2 A_{0}-4 A_{0} s n^{2}\left(-\frac{a \varepsilon \sqrt{A_{0}}}{p}(t+C), p\right)\right]^{\frac{1}{2}}
$$

which reduces to:

$$
x(t)=\frac{2 \varepsilon \sqrt{A_{0}}}{p}\left[1-p^{2} s n^{2}\left(-\frac{a \varepsilon \sqrt{A_{0}}}{p}(t+C), p\right)\right]^{\frac{1}{2}}
$$

Using the identity $k^{2} s n^{2}(z, k)+d n^{2}(z, k)=1$, the relation (27) becomes immediately:

$$
x(t)=\frac{2 \varepsilon \sqrt{A_{0}}}{p} d n\left(\frac{a \varepsilon \sqrt{A_{0}}}{p}(t+C), p\right)
$$

Knowing that $a=\varepsilon \sqrt{2 \beta}$, (28) may be written in the form:

$$
x(t)=\frac{2 \varepsilon \sqrt{A_{0}}}{p} d n\left(\frac{\sqrt{2 \beta A_{0}}}{p}(t+C), p\right)
$$

By making $A=\frac{2 \sqrt{A_{0}}}{p}$ and $\Omega=\frac{\sqrt{2 \beta A_{0}}}{p}$, that is $\Omega=\frac{A}{2} \sqrt{2 \beta}$, the general solution to (14) definitively reads:

$$
x(t)=\varepsilon \operatorname{Adn}(\Omega(t+C), p)
$$

where, $d n(z, k)$ is a Jacobian elliptic function and $p^{2}=\frac{2\left(\beta A^{2}+\omega_{0}^{2}\right)}{\beta A^{2}}$.

Figure 1 shows the comparison of (30) with the solution obtained by numerical integration. The solid line represents the behavior of solution (30) and circles depict the behavior of numerical solution with $\beta=3.95$, $\omega_{0}^{2}=8, A=2, x(t=0) 2$ and $\dot{x}(t=0)=0$.

Case 2: $\beta<0$ and $\omega_{0}^{2}>0$

For $\beta<0$ and $\omega_{0}^{2}>0$, it is also assumed that $-\frac{\omega_{0}^{2}}{\beta}$ $>2 A_{0}>0$. So, the general solution to the Duffing Equation 14 takes, using (29), the form:

$$
x(t)=\varepsilon A d c\left(\Omega(t+C), \sqrt{1-p^{2}}\right)
$$


where, $A=\frac{2 \sqrt{A_{0}}}{p}, \Omega=\frac{A}{2} \sqrt{2|\beta|}$. The function $d c(z, k)$ is a Jacobian elliptic function. In Fig. 2, the behavior of solution (31) is plotted with $\beta=-3, \omega_{0}^{2}=4, A=$ $1.3, x(t=0) 1.3$ and $\dot{x}(t=0)=0$.

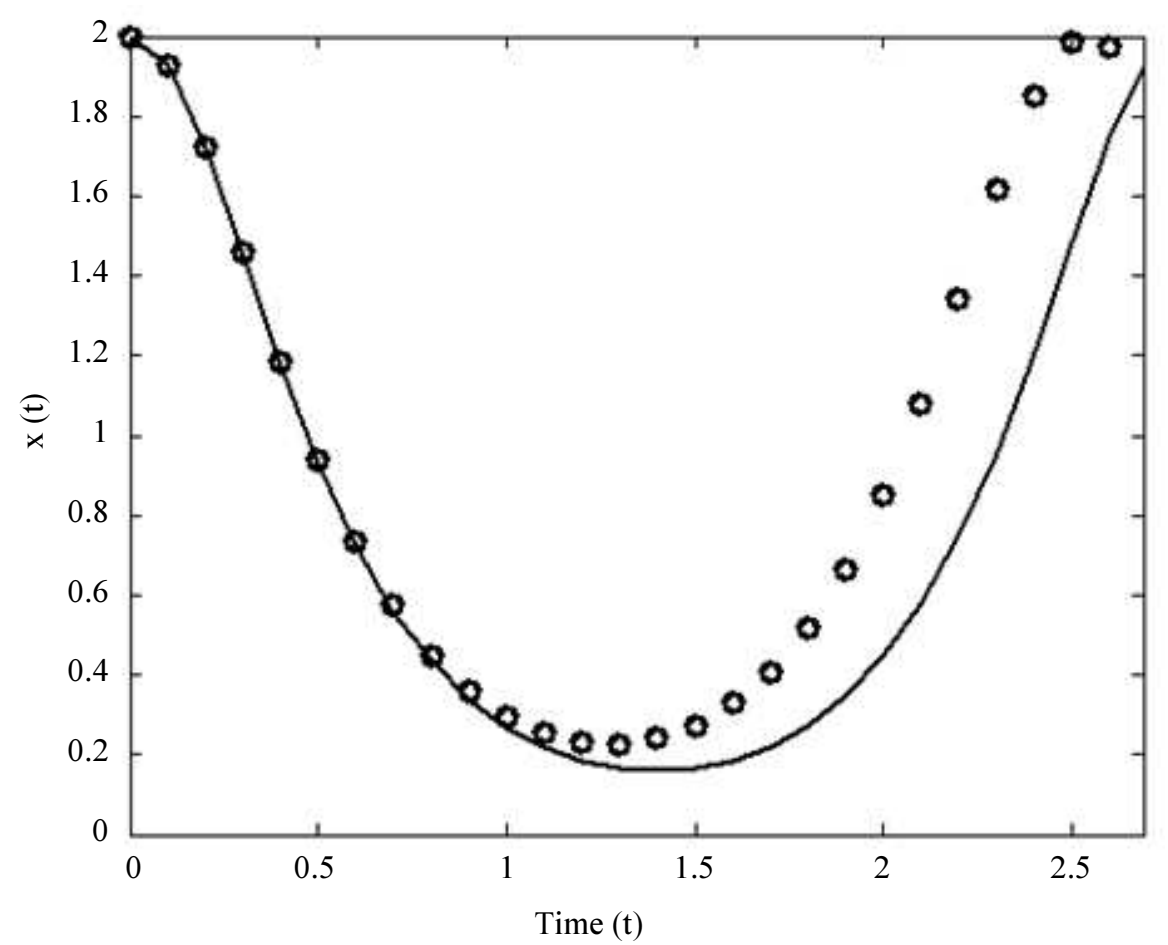

Fig. 1: Graphical comparison between the solution (30) in solid line and numerical solution in circles

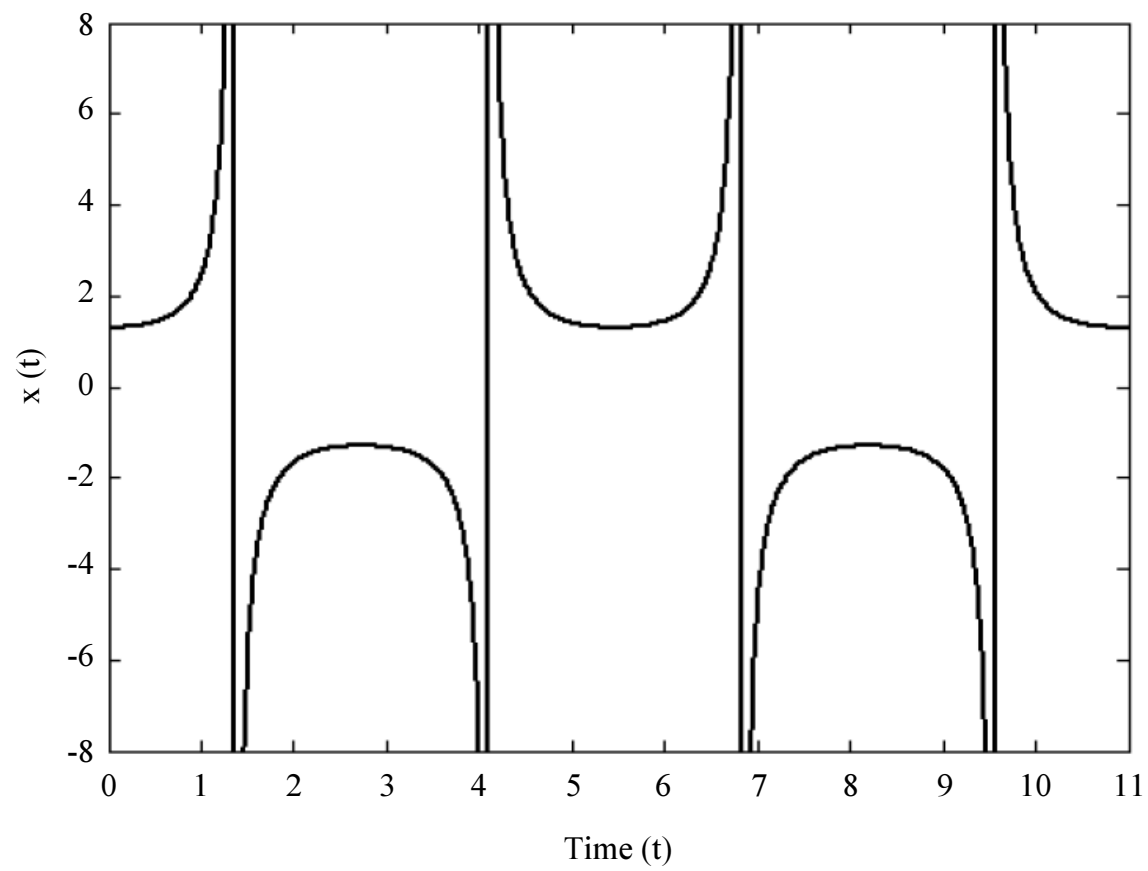

Fig. 2: Behavior of solution (31) 
Case 3: $\beta>0$ and $\omega_{0}^{2}>0$

In this case, it is assumed that $0<\left|-\frac{\omega_{0}^{2}}{\beta}\right|<2 A_{0}$, that is $0<\frac{\omega_{0}^{2}}{\beta}<2 A_{0}$. So, the integral $J$ may be written as (Gradshteyn and Ryzhik, 2007):

$$
J=-\frac{\sqrt{A_{0}}}{a A_{0}} F\left(\delta, \frac{1}{p}\right)
$$

where:

$$
\delta=\arcsin \left[\sqrt{\frac{2 \beta A_{0}(1-\sin (a \phi+\alpha))}{2 \beta A_{0}-\omega_{0}^{2}}}\right]
$$

$p$ has the preceding value and $F\left(\delta, \frac{1}{p}\right)$, denotes the elliptic integral of the first kind. In this regard the following relationship may be written, taking into consideration (19):

$$
\sqrt{\frac{2 \beta A_{0}(1-\sin (a \phi+\alpha))}{2 \beta A_{0}-\omega_{0}^{2}}}=s n\left[-a \varepsilon \sqrt{A_{0}}(t+C), \frac{1}{p}\right]
$$

that is:

$$
\sin (a \phi+\alpha)=1-\frac{2 \beta A_{0}-\omega_{0}^{2}}{2 \beta A_{0}} s n^{2}\left[-a \varepsilon \sqrt{A_{0}}(t+C), \frac{1}{p}\right]
$$

which may be rewritten:

$$
\sin (a \phi+\alpha)=1-\frac{2}{p^{2}} s n^{2}\left[-a \varepsilon \sqrt{A_{0}}(t+C), \frac{1}{p}\right]
$$

In this perspective the general solution to (14) reads:

$$
x(t)=\varepsilon \sqrt{\left[-\frac{\omega_{0}^{2}}{\beta}+2 A_{0}-\frac{4 A_{0}}{p^{2}} s n^{2}\left(-a \varepsilon \sqrt{A_{0}}(t+C), \frac{1}{p}\right)\right]}
$$

which may take the expression:

$$
x(t)=\frac{2 \varepsilon \sqrt{A_{0}}}{p} c n\left(a \sqrt{A_{0}}(t+C), \frac{1}{p}\right)
$$

Knowing $a=\varepsilon \sqrt{2 \beta}, A=\frac{2 \sqrt{A_{0}}}{p}$ and $\Omega=a \sqrt{A_{0}}$, that is $\Omega=\frac{p A \sqrt{2 \beta}}{2}$, or $\Omega=\sqrt{\beta A^{2}+\omega_{0}^{2}}$, the general solution to Duffing Equation 14 reduces immediately to the form:

$$
x(t)=\varepsilon A c n\left[\Omega(t+C), \frac{1}{p}\right]
$$

where, $\operatorname{cn}(z, k)$ denotes a Jacobian elliptic function. It can be noted that this result is in agreement with that found in (Lakshmanan and Rajaseekar, 2003). Figure 3 shows the comparison of solution (38) represented in solid line with the numerical solution represented in circles with $\beta=0.2, \omega_{0}^{2}=3.5, A=0.001, x(t=0)=0.001$ and $\dot{x}(t=0)=0$.

It would be now interesting to consider in the sequel of this work some Painlevé-Gambier type equations.

\section{Reduced Painlevé-Gambier XII Equation}

This subsection is intended to carry out the general solution to the Painlevé-Gambier XII equation (Ince, 1956):

$\dot{x}-\frac{\dot{x}^{2}}{x}-q x^{3}-\beta x^{2}-r-\frac{\delta}{x}=0$

for $r=\delta=0$, that is to say, to the reduced equation:

$\ddot{x}-\frac{\dot{x}^{2}}{x}-q x^{3}-\beta x^{2}=0$

The reduced Painlevé-Gambier Equation 40 may be found from (10) by choosing $\varphi(x)=\ln \left(x^{2}\right), \gamma=\frac{1}{2}, a^{2}=$ $-q$ and $c=\beta$. Therefore the general solution to (40) takes the expression:

$$
x(t)=A_{0} \sin (\varepsilon \sqrt{-q} \phi(t)+\alpha)-\frac{\beta}{q}
$$

where, $\varepsilon= \pm 1$ and $d t=\frac{d \phi(t)}{x(t)}$ that is:

$$
d t=\frac{d \phi(t)}{A_{0} \sin (\varepsilon \sqrt{-q} \phi(t)+\alpha)-\frac{\beta}{q}}
$$

By integration the quantity:

$$
J=\int \frac{d \phi(t)}{A_{0} \sin (\varepsilon \sqrt{-q} \phi(t)+\alpha)-\frac{\beta}{q}}
$$

leads to consider two distinct cases following the value of $\frac{q A_{0}}{\beta}$. 


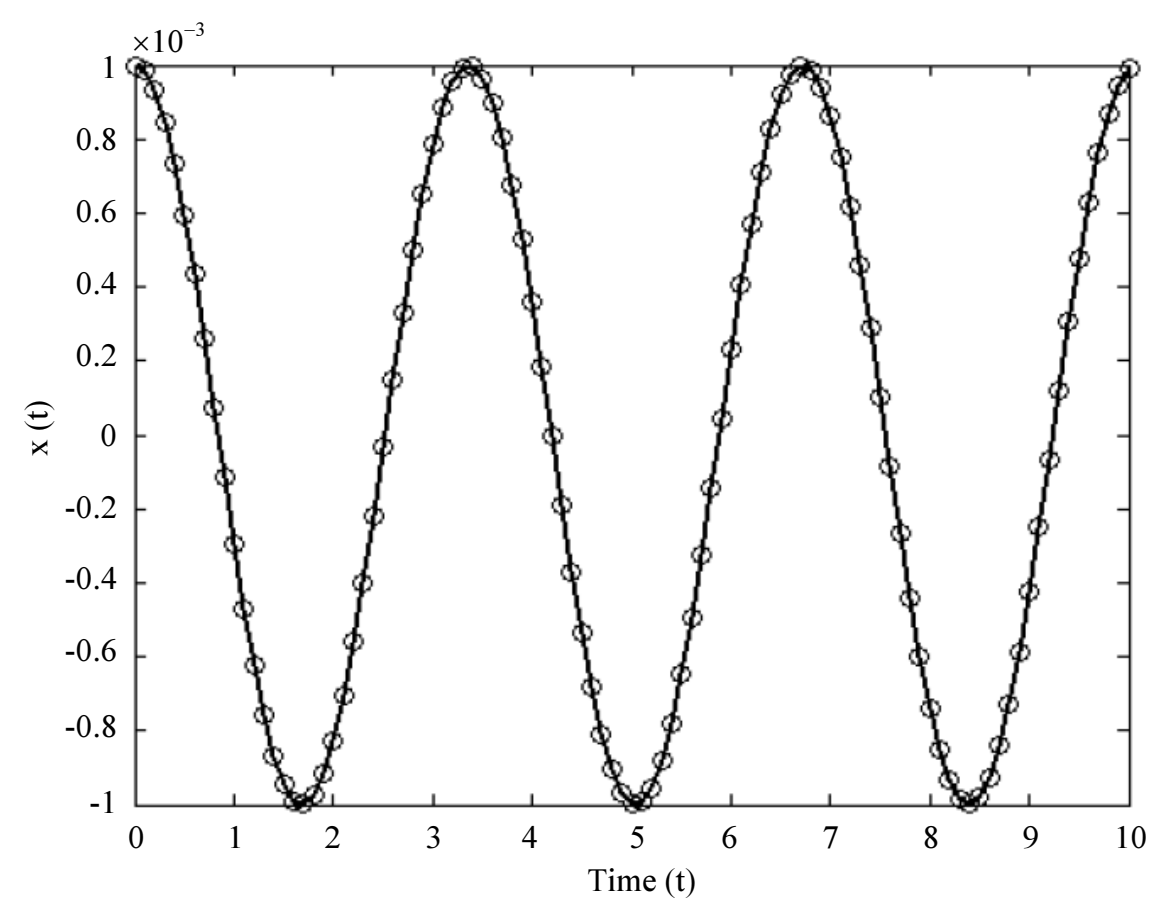

Fig. 3: Graphical comparison between the solution (38) in solid line and the numerical solution in circles

Case 1: $\frac{q^{2} A_{0}^{2}}{\beta^{2}}<1$

In this case the integral $J$ becomes (Gradshteyn and Ryzhik, 2007):

$$
J=-\frac{2 q}{\beta \varepsilon \sqrt{\frac{q^{3} A_{0}^{2}}{\beta^{2}}-q}} \operatorname{tg}^{-1}\left[\frac{\operatorname{tg}\left(\frac{\varepsilon \sqrt{-q} \phi(t)+\alpha}{2}\right)-\frac{q A_{0}}{\beta}}{\sqrt{1-\frac{q^{2} A_{0}^{2}}{\beta^{2}}}}\right]
$$

such that:

$$
\varepsilon \sqrt{-q} \phi(t)+\alpha=2 \operatorname{tg}^{-1}\left[\left(\sqrt{1-\frac{q^{2} A_{0}^{2}}{\beta^{2}}}\right) \Lambda+\frac{q A_{0}}{\beta}\right]
$$

where:

$$
\Lambda=\operatorname{tg}\left(-\frac{\beta \varepsilon \sqrt{\frac{q^{3} A_{0}^{2}}{\beta^{2}}}-q(t+C)}{2 q}\right)
$$

So the general solution to (40) becomes:

$x(t)=A_{0} \sin \left[2 \operatorname{tg}^{-1}\left[\left(\sqrt{1-\frac{q^{2} A_{0}^{2}}{\beta^{2}}}\right) \Lambda+\frac{q A_{0}}{\beta}\right]\right]-\frac{\beta}{q}$
The solution $x(t)$ is real for $q<0$. In this way, the solution (46) may clearly exhibit a harmonic periodic behavior with a shift factor $-\frac{\beta}{q}$.

Case 2: $\frac{q^{3} A_{0}^{2}}{\beta^{2}}>1$

This case corresponds to (Gradshteyn and Ryzhik, 2007):

$$
\begin{aligned}
& J=\left(-\frac{q}{\beta \varepsilon \sqrt{q-\frac{q^{3} A_{0}^{2}}{\beta^{2}}}}\right) \times \\
& \ln \left[\frac{\operatorname{tg}\left(\frac{\varepsilon \sqrt{-q} \phi(t)+\alpha}{2}\right)-\frac{q A_{0}}{\beta}-\sqrt{q-\frac{q^{3} A_{0}^{2}}{\beta^{2}}}}{\operatorname{tg}\left(\frac{\varepsilon \sqrt{-q} \phi(t)+\alpha}{2}\right)-\frac{q A_{0}}{\beta}+\sqrt{q-\frac{q^{3} A_{0}^{2}}{\beta^{2}}}}\right]
\end{aligned}
$$

which gives according to (42):

$$
\begin{aligned}
& \varepsilon \sqrt{-q} \phi(t)+\alpha= \\
& 2 \operatorname{tg}^{-1}\left[\frac{\left(\sqrt{\frac{q^{2} A_{0}^{2}}{\beta^{2}}-1}\right) \Lambda_{1}}{\Lambda_{2}}+\frac{q A_{0}}{\beta}\right]
\end{aligned}
$$


with:

$$
\Lambda_{1}=1+\exp \left(-\frac{\beta \varepsilon}{q} \sqrt{q-\frac{q^{3} A_{0}^{2}}{\beta^{2}}}(t+C)\right)
$$

and:

$$
\Lambda_{2}=1-\exp \left(-\frac{\beta \varepsilon}{q} \sqrt{q-\frac{q^{3} A_{0}^{2}}{\beta^{2}}}(t+C)\right)
$$

Therefore the general solution $x(t)$ may be expressed in the form:

$$
x(t)=A_{0} \sin \left[2 \operatorname{tg}^{-1}\left[\xi+\frac{q A_{0}}{\beta}\right]\right]-\frac{\beta}{q}
$$

with:

$$
\xi=\frac{\sqrt{\frac{q^{2} A_{0}^{2}}{\beta^{2}}-1}\left(1+\exp \left(-\frac{\beta \varepsilon}{q} \sqrt{q-\frac{q^{3} A_{0}^{2}}{\beta^{2}}}(t+C)\right)\right)}{1-\exp \left(-\frac{\beta \varepsilon}{q} \sqrt{q-\frac{q^{3} A_{0}^{2}}{\beta^{2}}}(t+C)\right)}
$$

For $q<0$, the solution (49) is real and then may exhibit also a harmonic periodic behavior with a shift factor $-\frac{\beta}{q}$.

Consider now as final illustrative example a generalized equation which contains the PainlevéGambier XIX equation as special case.

\section{Generalized Equation}

The Painlevé-Gambier XIX equation is of the form (Ince, 1956):

$\ddot{x}-\frac{1}{2} \frac{\dot{x}^{2}}{x}-4 x^{2}-2 x=0$

A general form of (50) may be written as:

$\ddot{x}-\frac{1}{2} \frac{\dot{x}^{2}}{x}+a^{2} x^{2}-c x=0$

So that for $a^{2}=-4$ and $c=2$, one may recover the Painlevé-Gambier XIX equation. The equation (51) may be obtained from (10) by setting $\gamma=\frac{1}{4}$ and $\varphi(x)=\ln \left(x^{2}\right)$. Thus the solution to (51) immediately takes the expression following (12):

$$
x(t)=A_{0} \sin (a \phi(t)+\alpha)+\frac{c}{a^{2}}
$$

where:

$$
d t=\frac{d \phi(t)}{\sqrt{A_{0} \sin (a \phi(t)+\alpha)+\frac{c}{a^{2}}}}
$$

The equation is of the same form as (19). So, three distinct cases may be distinguished.

Case 1: $a^{2}>0, c>0$ and $\frac{c}{a^{2}}>A_{0}>0$.

In this case:

$$
p=a \sqrt{\frac{2 A_{0}}{a^{2} A_{0}+c}}
$$

and:

$$
\delta=\arcsin \left(\sqrt{\frac{1-\sin (a \phi+\alpha)}{2}}\right)
$$

so the integral:

$$
J=\int \frac{d \phi(t)}{\sqrt{A_{0} \sin (a \phi(t)+\alpha)+\frac{c}{a^{2}}}}
$$

becomes:

$$
J=-\frac{p \sqrt{2}}{a \sqrt{A_{0}}} F(\delta, p)
$$

where, $F(\delta, p)$ designates the elliptic integral of the first kind. So according to (53) one may write:

$$
-\frac{a \sqrt{A_{0}}}{p \sqrt{2}}(t+C)=F(\delta, p)
$$

that is:

$$
\sin \delta=s n\left(-\frac{a \sqrt{2 A_{0}}}{2 p}(t+C), p\right)
$$

In this regard, the general solution (52) becomes:

$$
x(t)=\left(\frac{2 A_{0}}{p^{2}}\right) d n^{2}\left(\frac{a \sqrt{2 A_{0}}}{2 p}(t+C), p\right)
$$


which may take the expression:

$x(t)=A^{2} d n^{2}(\Omega(t+C), p)$

where, $A=\frac{\sqrt{2 A_{0}}}{p}$ and $\Omega=\frac{a \sqrt{2 A_{0}}}{2 p}$ that is $\Omega=\frac{a A}{2}$ and $p$ may be rewritten as $p^{2}=\frac{2\left(a^{2} A^{2}-c\right)}{a^{2} A^{2}}$.

Case 2: $a^{2}<0, c>0$ and $0<\left|\frac{c}{a^{2}}\right|<A_{0}$

In such a situation, the integral $J$ becomes:

$J=-\frac{1}{a} \sqrt{\frac{2}{A_{0}}} F\left(\delta, \frac{1}{p}\right)$

where, $\quad \delta=\arcsin \left(\sqrt{\frac{a^{2} A_{0}(1-\sin (a \phi+\alpha))}{a^{2} A_{0}+c}}\right)$ and $p=\sqrt{\frac{2 a^{2} A_{0}}{a^{2} A_{0}+c}}$. So the use of (53) leads to:

$-a \sqrt{\frac{A_{0}}{2}}(t+C)=F\left(\delta, \frac{1}{p}\right)$

which may give:

$\sqrt{\frac{a^{2} A_{0}(1-\sin (a \phi+\alpha))}{c+a^{2} A_{0}}}=s n\left[-a \sqrt{\frac{A_{0}}{2}}(t+C), \frac{1}{p}\right]$

from which:

$\sin (a \phi+\alpha)=1-\frac{c+a^{2} A_{0}}{a^{2} A_{0}} s^{2}\left(-a \sqrt{\frac{A_{0}}{2}}(t+C), \frac{1}{p}\right)$

Therefore the general solution (52) may take the expression:

$$
x(t)=\frac{2 A_{0}}{p^{2}}\left[1-s n^{2}\left(-a \sqrt{\frac{A_{0}}{2}}(t+C), \frac{1}{p}\right)\right]
$$

The general solution (65) may be also expressed as:

$x(t)=\frac{2 A_{0}}{p^{2}} c n^{2}\left(-a \sqrt{\frac{A_{0}}{2}}(t+C), \frac{1}{p}\right)$

which becomes:

$x(t)=A^{2} c n^{2}\left(\Omega(t+C), \frac{1}{p}\right)$ where $A^{2}=\frac{2 A_{0}}{p^{2}}$.

For $a^{2}=i^{2}\left|a^{2}\right|$, that is $a= \pm i \sqrt{\left|a^{2}\right|}$, the general solution $x(t)$ may take the form:

$$
x(t)=A^{2} c n^{2}\left(i \frac{\sqrt{\left|a^{2}\right|}}{2} p A(t+C), \frac{1}{p}\right)
$$

that is:

$$
x(t)=\frac{A^{2}}{c n^{2}\left(\frac{\sqrt{\left|a^{2}\right|}}{2} p A(t+C), \sqrt{1-\frac{1}{p^{2}}}\right)}
$$

which becomes definitively:

$$
x(t)=\frac{A^{2}}{c n^{2}\left(\Omega(t+C), \sqrt{1-\frac{1}{p^{2}}}\right)}
$$

where, $\Omega=\frac{\sqrt{\left|a^{2}\right|}}{2} p A$ and $i$ is the purely imaginary number.

By making $a^{2}=-4$ and $c=2$, the exact doubly periodic solution to Painlevé-Gambier XIX equation may be written as:

$$
x(t)=\frac{A^{2}}{c n^{2}\left(\sqrt{2 A^{2}+1}(t+C), \sqrt{\frac{A^{2}+1}{2 A^{2}+1}}\right)}
$$

that is:

$$
x(t)=\frac{A^{2}}{c n^{2}\left(\Omega(t+C), \sqrt{\frac{A^{2}+1}{2 A^{2}+1}}\right)}
$$

where $A^{2}=\frac{2 A_{0}-1}{2}$ and $p=\frac{1}{A} \sqrt{2 A^{2}+1}$.

Case 3: $a^{2}>0, c<0$ and $0<\left|\frac{c}{a^{2}}\right|<A_{0}$ This case corresponds also to $p=\sqrt{\frac{2 a^{2} A_{0}}{a^{2} A_{0}+c}}$, $\delta=\arcsin \left(\sqrt{\frac{a^{2} A_{0}(1-\sin (a \phi+\alpha))}{a^{2} A_{0}+c}}\right)$ and the integral $J$ becomes: 


$$
J=-\frac{1}{a} \sqrt{\frac{2}{A_{0}}} F\left(\delta, \frac{1}{p}\right)
$$

So the general solution (52) may be written as:

$$
x(t)=\frac{2 A_{0}}{p^{2}} c n^{2}\left(a \sqrt{\frac{A_{0}}{2}}(t+C), \frac{1}{p}\right)
$$

that is:

$$
x(t)=A^{2} c n^{2}\left(\Omega(t+C), \frac{1}{p}\right)
$$

where:

$$
A^{2}=\frac{2 A_{0}}{p^{2}}
$$

and:

$$
\Omega=\frac{a p A}{2}
$$

The parameter $p$ may also be expressed as $p^{2}=\frac{2\left(a^{2} A^{2}-c\right)}{a^{2} A^{2}}$.

That being so it is then possible to show the equivalence between the Duffing equation and the generalized Equation 51.

\section{Equivalence between Equations}

This section is devoted to highlight the mathematical equivalence between the Duffing equation and the generalized Equation 51. The comparison of (19) with (53) as well as the comparison of resulting general solutions suggest this mathematical equivalence, that is to say the mapping of the Duffing equation onto the generalized Equation 51 and vice versa. In other words, the general solution to the Duffing equation may be obtained in terms of the solution to the generalized Equation 51 and vice versa. Formally consider the variable transformation:

$$
x^{2}=2 w
$$

due to the above general solutions to Duffing equation and general solutions to the generalized Equation 51.

The substitution of (77) into (14) yields:

$$
\ddot{w}-\frac{1}{2} \frac{\dot{w}^{2}}{w}+4 \beta w^{2}+2 \omega_{0}^{2} w=0
$$

which is the generalized Equation 51 by taking $a^{2}=2 \beta$ and $c=-2 \omega_{0}^{2}$. So with that the equivalence between Duffing equation and the generalized Equation 51 has been shown and a discussion may be formulated for the present work.

\section{Discussion}

In the theory of nonlinear differential equations, the problem of calculating explicit and exact general periodic solutions often arises acutely, given the nonexistence of a master solving method. In this study, it was proposed to check the efficiency of a generalized Sundman transformation supposed to solve certain types of nonlinear second order differential equations. Thus, the well known cubic Duffing equation in various branches of physics such as classical and quantum mechanics has been chosen as an illustration of the proposed linearization transformation given, to our knowledge, that a generalized Sundman transformation has never been used to calculate its explicit and exact general periodic solutions. It is well known that all the twelve Jacobian elliptic functions are solutions of this equation (Schwalm, 2015). By application of the proposed linearization transformation, explicit and exact general periodic solutions of the cubic Duffing equation were computed, as expected, as Jacobian elliptic functions with simplicity and elegance in the solution steps, which characterize the generalized Sundman transformation. In this perspective, explicit and exact general periodic solutions of Painlevé-Gambier XIX equation have been computed for the first time using a generalized Sundman transformation, as Jacobian elliptic functions. On the other hand, it was possible to calculate explicit and exact general trigonometric solutions but with a shift factor to a reduced Painlevé-Gambier XII equation for the first time in accordance with a suitable parametric choice. With these convincing results, it would be possible now to conclude this research contribution.

\section{Conclusion}

The vital problem of finding explicit and exact general periodic solutions to nonlinear differential equations is still an active research field of mathematics. In this study, explicit and exact general periodic solutions are computed for various types of Liénard equations by means of a generalized Sundman linearization approach. In this way, the explicit and exact general solutions to the cubic Duffing equation as well as for some Painlevé- Gambier type equations are in a straightforward manner determined. In so doing, it has been demonstrated for the first time that for suitable parametric choice a reduced Painlevé-Gambier XII equation exhibits trigonometric solutions but with a shift factor, which is a major finding in the investigation of 
the analytical properties of the Painlevé-Gambier equations. It is also for the first time that explicit and exact general periodic solutions have been calculated for Painlevé-Gambier XIX equation by a generalized Sundman transformation as Jacobian elliptic functions. Thus it has been shown that the generalized Sundman transformation has a great advantage over numerous methods and can be a powerful mathematical tool in the determination of explicit and exact general solutions to the problems of physics and applied sciences which are described in terms of nonlinear differential equations.

\section{Author's Contributions}

All authors contributed to the development and formulation of this work. All authors read and approved the final manuscript for publication.

\section{Ethics}

The authors declare that there exists no competing interests.

\section{References}

Akande, J., D.K.K. Adjaï, L.H. Koudahoun, Y.J.F. Kpomahou and M. D. Monsia, 2017a. Theory of exact trigonometric periodic solutions to quadratic Liénard type equations. viXra: 1704.0199 V3.

Akande, J., D.K.K. Adjaï, L.H. Koudahoun, B. Rath and P. Mallick et al., 2017b. Exact quantum mechanics of quadratic Liénard type oscillator equations with bound states energy spectrum. viXra: $1702.0242 \mathrm{~V} 1$.

Euler, N. and M. Euler, 2004. Sundman symmetries of nonlinear second-order and third-order ordinary differential equations. J. Nonlinear Math. Phys., 11: 399-421. DOI: 10.2991/jnmp.2004.11.3.9

Gradshteyn, I.S. and I.M. Ryzhik, 2007. Table of Integrals, Series and Products. 7th Edn., Academic Press, ISBN-10: 0123736374, pp: 1200.

Ince, E.L., 1956. Ordinary differential equations. Universal Library, Dover, New York.
Kerschen, G., K. Worden, A.F. Vakakis and J.C. Golinval, 2006. Past, present and future of nonlinear system identification in structural dynamics. Mech. Syst. Signal Process., 20: 505-592. DOI: $10.1016 /$ j.ymssp.2005.04.008a

Kpomahou, Y.J.F. and M.D. Monsia, 2015. Hardening nonlinearity effects on forced vibration of viscoelastic dynamical systems to external step perturbation field. Int. J. Adv. Applied Math. Mech., 3: 16-32.

Lakshmanan, M. and S. Rajaseekar, 2003. Nonlinear Dynamics: Integrability, Chaos and Patterns. 1st Edn., Springer-Verlag, Berlin, ISBN-10: 8181280121 .

Monsia, M.D., 2012. A mathematical model for predicting the relaxation of creep strains in materials. Phys. Rev. Res. Int., 2: 107-124.

Nakpim, W. and S.V. Meleshko, 2010. Linearization of second-order ordinary differential equations by generalized Sundman transformations. SIGMA, 6: $1-11$.

Nayfeh, A.H. and D.T. Mook, 1979. Nonlinear Oscillations. 1st Edn., John Wiley and Sons, New York, ISBN-10: 0471035556, pp: 720.

Partha, G., B. Khanra and A.G. Choudoury, 2010. On generalized Sundman transformation method, first integrals, symmetries and solutions of equations of Painlevé-Gambier type. Nonlinear Anal., 72: 3247-3257. DOI: 10.1016/j.na.2009.12.004

Reinhardt, W.P. and P.L. Walker, 2010. Jacobian Elliptic Functions. In: NIST Handbook of Mathematical Function, Franck, W.J.O., D.W. Lozier, R.F. Boisvert and C.W. Clark (Eds.), Hardback, ISBN13: 978-0-521-19225-5, pp: 550-567.

Schwalm, W., 2015. Lectures on Selected Topics in Mathematical Physics: Elliptic Functions and Elliptic Integrals. 1st Edn., Morgan and Claypool Publishers, ISBN-10: 1681740389, pp: 67.

Utz, W.R., 1970. Periodic solutions of a nonlinear second order differential equation. SIAM J. Applied Math., 19: 56-59. DOI: 10.1137/0119005 